\title{
Functionalization of Graphene and Reduced Graphene Oxide in Different Matrices
}

\author{
E. Celasco ${ }^{1,2, *}$, M. Sangermano ${ }^{3}$ \\ ${ }^{I}$ Dipartimento di Fisica Università di Genova, Via Dodecaneso 33, 16146, Italy \\ ${ }^{2}$ CNR IMEM Unità Operativa di Genova, Via Dodecaneso 33, 16146, Genova, Italy \\ ${ }^{3}$ Politecnico di Torino, Applied Science and Technology Department, Corso Duca degli Abruzzi 24, 10129 Torino, Italy
}

*Corresponding author: Tel: (+39) 010353 6409; E-mail: celasco@ fisica.unige.it

Received: 08 August 2018, Revised: 27 September 2018 and Accepted: 07 January 2019

DOI: $10.5185 /$ amlett.2019.2201

www.vbripress.com/aml

\begin{abstract}
Graphene $(\mathrm{G})$ presents a huge variety of intriguing properties, as extraordinary electronic transport characteristics. $\mathrm{G}$, thanks to its low chemical reactivity, can also be used as an active support for catalytic nanoparticles. Some possible graphene application could be: its employment in active material in electronic devices such as sensors [1], batteries [2], supercapacitors, hydrogen storage systems or as fillers to produce multifunctional nanocomposite polymeric materials [3]. In more detail we would like to examine: different approach of reduction and functionalization of in situ reduced graphene oxide obtaining an enhancement of thermal conductivity and an resistivity decrease [4]. Surface modification and functionalization of rGO to improve its dispersion in organic solvent and also polymeric matrix [5]. Copyright (C) VBRI Press.
\end{abstract}

Keywords: Graphene, reduced graphene oxide, surface chemistry.

\section{Introduction}

Graphene is one of the most studied material according hits intruing electrical and chemical characteristics. It presents an exceptional electron mobility at room temperature $\left(2.5 \cdot 10^{5} \mathrm{~cm}^{2} \mathrm{~V}^{-1} \mathrm{~s}^{-1}\right)$, high thermal conductivity $\left(5000 \mathrm{Wm}^{-1} \mathrm{~K}^{-1}\right)$, and mechanical properties [6-8] which make it particularly appealing for a wide range of applications.

Recently the scientific community develops its interest in polymeric matrix with reduced Graphene Oxide dispersed in, to improve the conductivity and the characteristics of the polymeric matrix. Actually, the study of high performing polymeric nanocomposite give the possibility to explore new properties of the carbon based materials. One crucial step will be the surface modification of low-cost graphene oxide in the matrix of this polymer.

In this paper a comparison of two different methods to obtain conductive and functionalized polymers will be described, with their relative advantages and limits. The two principles are different, in the first method we will describe an electrically conductive epoxy resin composite exploiting in-situ reduction of graphene oxide GOx during epoxy thermal curing in presence of amine.

The novelty of this first method consists in its easy way to scale one step process finalized to obtain conductive epoxy cured materials with well dispersed GOx reduced during the treatment.
In the second method we will explore a tool for graphene oxide reduction by Ultra Violet (UV) technique and two fast steps of functionalization. The advantage of this second method is the whole process at room temperature, with the direct consequence of a wider range of application especially on all kind of materials with possible degradation and modification with temperature.

\section{Experimental}

The epoxy resin is formed by: Theepoxyresin bisphenol-A-diglycidyl ether (DGEBA, Mw=340.41 g $\mathrm{mol}^{-1}$ and the amine tetraethylpentamine (TEPA, $\mathrm{Mw}=189.302 \mathrm{~g} \mathrm{~mol}^{-1}$ ) from Aldrich, is employed. GOx from CheapTubes (USA). Tetrahydrofurane (THF, from Aldrich) was used as the solvent. The epoxy-rGOx nanocomposite is prepared dispersing GOx into the THF polar solvent by ultra-sonication for $30 \mathrm{~min}$.

The epoxy resin was subsequently added to the THF and the dispersion homogenized by means of an Ultraturrax mixer at $30000 \mathrm{rpm}$ for $15 \mathrm{~min}$. At the end of this process the resulting formulation was degassed under vacuum for one night, then the adequate amount of TEPA added and the formulation stirred, poured into a mould and cured for $2 \mathrm{~h}$ at $80^{\circ} \mathrm{C}$. The crosslinked sample was then subjected to a post-curing treatment at $150^{\circ} \mathrm{C}$ for $30 \mathrm{~min}$.

For the functionalization reduced graphene oxide the materials employed are: 
Graphene Oxide (GO) with thickness range form $0.7-$ to $1.2 \mathrm{~nm}$, from Cheap Tubes Inc. (USA) Benzophenone (BP) (Sigma-Aldrich) is used as anchoring agent. Poly (ethylene glycol) methacrylate PEGMA (Mw 475, Sigma-Aldrich), perfluoro butyl acrylate PFBA (Daikin Chemicals), and 2(Dimethylamino) ethyl methacrylate DMAEM (Sigma-Aldrich) are used to functionalize the reduced GO. And finally Dimethylformamide DMF and Ethanol is used as solvents.

The characterization employed for these experiments are:

X-rays photoelectron spectroscopy (XPS) of versa Probe 5000 of PHI Electronics Company. The graphene solution for the XPS is homogeneously spinned on silicon wafer. The scans are acquired with a monochromatic. X-ray beam (Al K-alpha line at $1486.6 \mathrm{eV}$ ) and with a double beam neutralization charging system, consisting by an ion gun combined with an electron gun. Thermal analysis is carried on with a Mettler-Toledo DSC equipped with a robotic $\mathrm{arm}$. Samples having masses of approximately $10 \mathrm{mg}$ were cured in aluminium pans with pierced lids in a nitrogen atmosphere after the equipment calibration with indium standards. The DSC was used to study isothermal curing at Different temperatures. Infrared spectroscopy is done using a Thermo-Nicolet 5700 instrument equipped with an attenuated total reflectance (ATR) expansion tool.

\section{Results and discussion}

The first method developed is the in situ reduction of graphene oxide (GOx) during the epoxy thermal curing in presence of amine. One direct and more accurate chemical characterization in order to obtain a direct evidence of reduction mechanism from GO to rGO is the $\mathrm{X}$ - ray photoemission spectroscopy.

With this surface sensitive characterization, we have the opportunity to compare the starting point GO sample, crosschecking simultaneously the presence of amine, and the result of the thermal reduction step due to the presence of amine.

In Fig. 1 is monitored the drastically reduction of carboxylic group at $287 \mathrm{eV}$ and $288 \mathrm{eV}$, after the thermal treatment. The first step of XPS analyses is the survey scan a complete scan from 0 to $1200 \mathrm{eV}$ (not shown). With this complete overview, it is possible to monitor all elements present on the sample excluding eventual external contamination.

We found evidence of Carbon 1s peak (around $285 \mathrm{eV})$ Oxygen 1s peak $(530 \mathrm{eV})$ and Nitrogen $1 \mathrm{~s}$ peak (at $400 \mathrm{eV}$ ) and no evidence of contaminant. The chemical bonds present on the samples are reported in the high resolution XPS spectra of Fig. 1a and Fig. 1b.

In both panels are represented C-C bond, at $284,7 \mathrm{eV}$ the main peak $\mathrm{C}=\mathrm{O}$ and $\mathrm{C}-\mathrm{N}$ at $287 \mathrm{eV}$ and the last one relative to $\mathrm{O}-\mathrm{C}=\mathrm{O}$ at $288 \mathrm{eV}$. The same peaks are present in the panel $\mathrm{b}$ ) but the dominant one is the $\mathrm{C}-\mathrm{C}$ one while the $\mathrm{C}=\mathrm{O}$ presents a huge reduction. This behaviour is due and confirms to the reduction of the sample after the treatment. In a semiquantitative way the $\mathrm{C}=\mathrm{O} / \mathrm{C}-\mathrm{C}$ ratio of the panel a) gives $89 \%$ value respect the $7 \%$ of the same ratio in the panel b). This values confirms us that a good reduction treatment occurs.
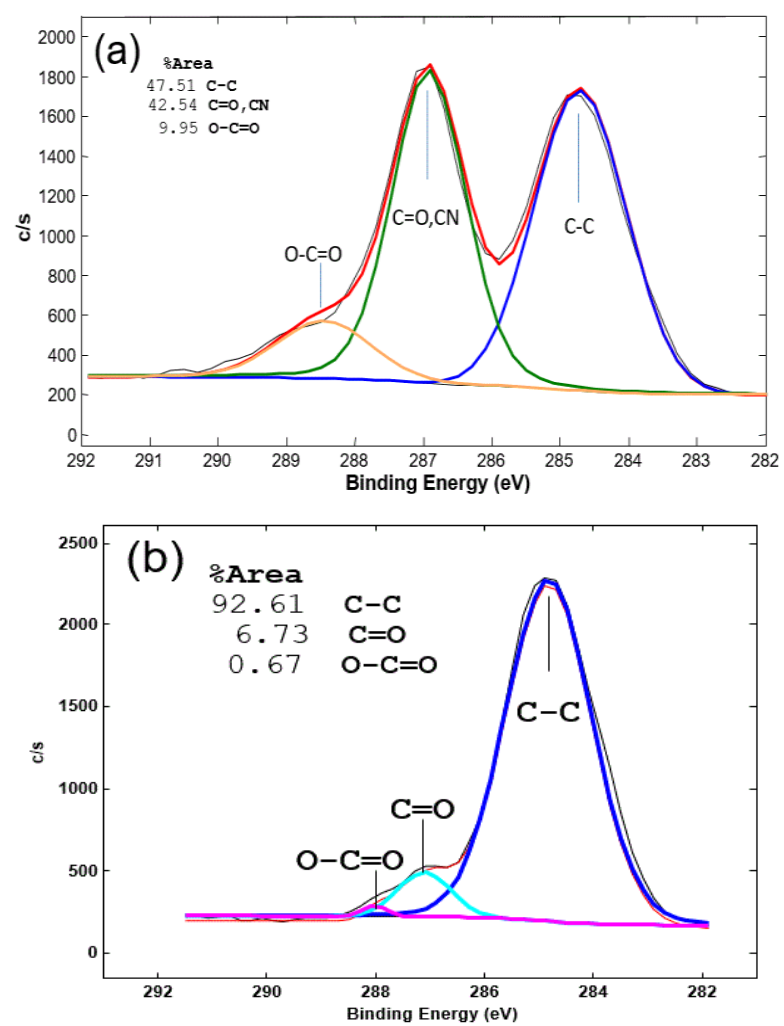

Fig. 1. High resolution XPS (C1s region) of (a) GOx starting sample resin in the presence of amine. (b) Reduced sample in the presence of $20 \mathrm{phr}$ TEPA at $80^{\circ} \mathrm{C}$.

In order to optimize the best curing condition DSC-dynamic analyses, varying the amine concentration, is performed. The main result stressed in Fig. 2 where is reported the evolved heat during DSC-dynamic analyses of DGEBA containing TEPA. The top spectrum is relative to 20 part per hundred (phr) amine, while 15 and $10 \mathrm{phr}$ is respectively for the middle end the bottom spectra.

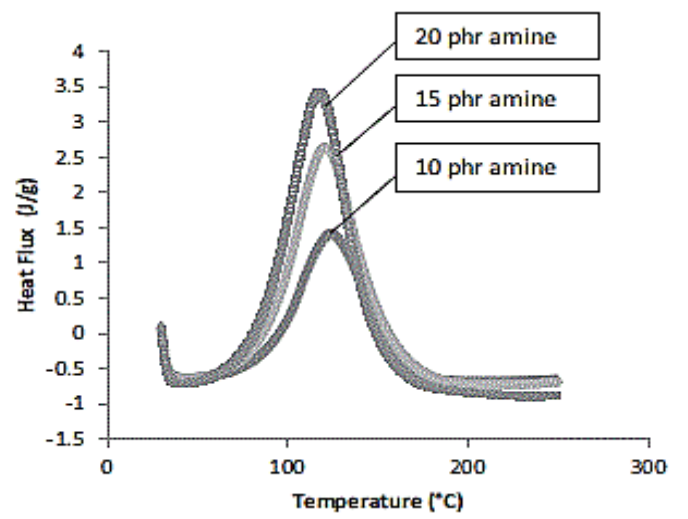

Fig. 2. DSC-dynamic analyses of DGEBA containing TEPA in the range between 10 to $20 \mathrm{phr}$. 
Assuming that the heat evolved during the curing process is generated by the disappearance of epoxide groups the area underneath the exothermic peak can be used to determine the fractional conversion of the epoxy resin. From this analyses is evident that the best condition is the one with $20 \mathrm{phr}$ amine. The best curing conditions for the pristine epoxy resin: $20 \mathrm{phr}$ of the TEPA hardener and curing time of $2 \mathrm{~h}$ at $80^{\circ} \mathrm{C}$. Adding GOx during curing condition have the consequence of improve the reactivity due to the much larger thermal conductivity for the filled composition compared to the pristine resin. One direct consequence is an enhancement on thermal activation of the epoxy crosslinking activation.

The last characterization on this system is the electrical characterization on different composite sample. The unfilled sample presents a noisy signal with a not conductive trend (about $10^{9} \mathrm{ohm} \mathrm{cm}$ ). While the sample with $2 \mathrm{phr}$ GOx have a resistivity of $10^{7} \mathrm{ohm} \mathrm{cm}$ with ohmic behaviour. Increasing the GOx to $6 \mathrm{phr}$ the results is a decrease of resistivity of one order of magnitude, but with a diode like behaviour. A second method described here is a useful tool for reduce polymer with GO, with a two functionalization steps.

The advantage of this second method is avoid thermal treatment increasing the possible application of this polymeric conductive material. The functionalization process consists in $10 \mathrm{mg}$ of GO in DMF $(0.5 \mathrm{mg} / \mathrm{ml}$ solution $)$ placed in a $100 \mathrm{ml}$ three necked flask. The obtained mixture was sonicated in an ultrasound bath until a homogeneous dispersion was achieved. The second step consist to add $30 \mathrm{mg}$ of BP powder to the solution, after magnetic stirred step and degas process by bubbling with nitrogen for 30 min. The final mixture was UV irradiated with a high-pressure mercury lamp $\left(40 \mathrm{~mW} / \mathrm{cm}^{2}\right.$ intensity) while stirring at room temperature for $5 \mathrm{~min}$.

The solution was finally transferred into centrifuge tubes and centrifuged $5000 \mathrm{rpm}$ of speed, for $10 \mathrm{~min}$. The precipitates were washed with ethanol and centrifuged several times in order to remove the unreacted BP. The purified product was dried overnight at $60^{\circ} \mathrm{C}$. The second functionalization step consists in the dispersion of $10 \mathrm{mg}$ of the modified powder in $20 \mathrm{ml}$ of DMF in the presence of $50 \mathrm{mg}$ of the desired acrylic monomer or oligomer. The final solution was magnetically stirred, degassed by bubbling with nitrogen for $30 \mathrm{~min}$ and subsequently UV irradiated for different times. The mixture was then centrifuged, washed and dried. In the Fig. 3 is represented a schematic view of the whole process.

Semipinacol radicals is formed after UV irradiation of BP in DMF solution. The obtained semipinacol could recombine, creating a covalent bond, with the radicals of the GO sheets. During this process GO after a light-induced reduction [9-11] reduce itself in RGO. The RGO has desirable properties like electrical and thermal conductivity, but unfortunately presents the big limitation to has a poor solubility in polymers [7]. After a second UV irradiation the bond between the semipinacol and surface GO is broken homolytically. The UV irradiaton [12] allows the generation of starting point for the free radical polymerization in presence of suitable monomers.

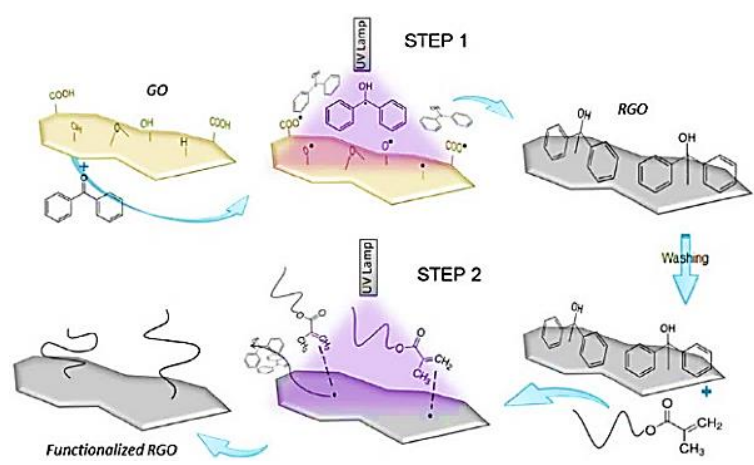

Fig. 3. Schematic layout of the two steps functionalization reaction.
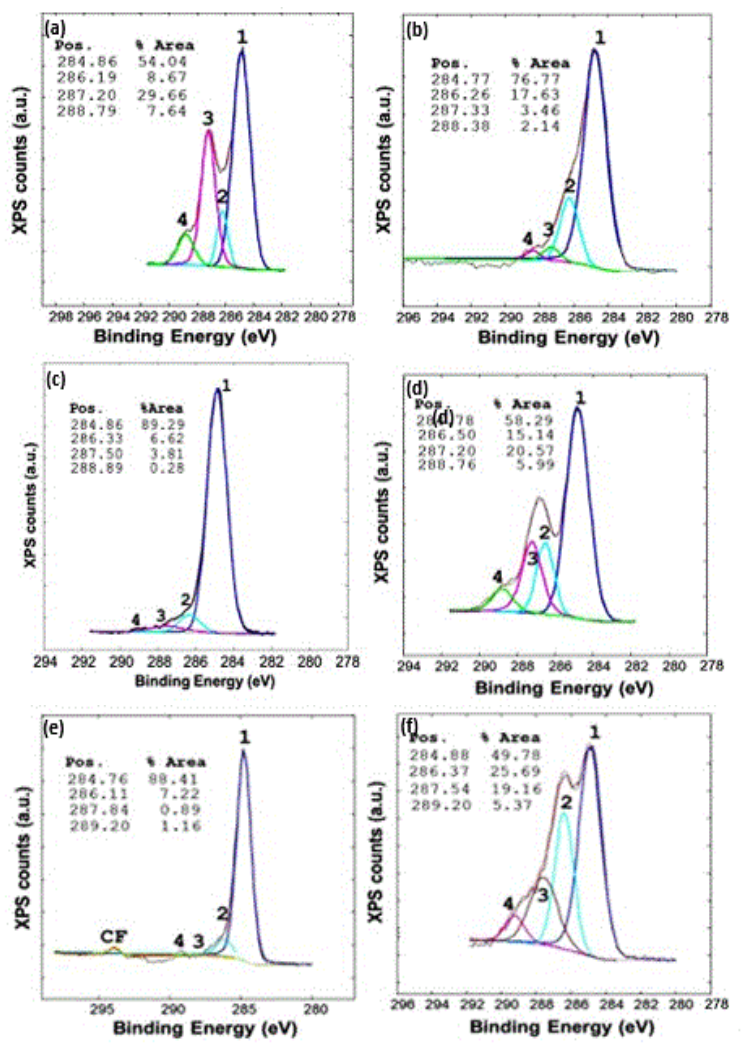

Fig. 4. XPS spectra of C1s of GO (panel a), GO after $5 \mathrm{~min}$ of UV irradiation (panel b), GO after 5min of UV irradiation in the presence of BP (panel c), RGO/PEGMA (d), RGO/PFBA (panel e), RGO/DMAEM (panel f).

With this method it is possible to obtain a polymer-functionalized RGO that presents the intruing properties of graphene with improved compatibility with polymer matrices. An almost real time cross check characterization to monitor the quality of obtained reduction reaction and functionalization is the XPS analyses. In the Fig. 4 is reported a complete characterization of the whole 
process. XPS is one of the most surface sensitive analysis, it gives us a compositional information. Fig. 4 is divided in 6 panels: XPS spectra of (a) $\mathrm{C} 1 \mathrm{~s}$ of $\mathrm{GO}$, (b) $\mathrm{GO}$ after 5 min of UV irradiation, (c) GO after 5 min of UV irradiation in the presence of BP, (d) RGO/PEGMA, (e) RGO/PFBA, (f), RGO/DMAEM.

The fitting procedure $[13,14]$ giving us also a semi-quantitative information on the oxidation/reduction state of the surface after oxidative/reduction treatments. The $\mathrm{C}-\mathrm{C}$ bonds is labelled in the figure and in the following with the number $1, \mathrm{C}-\mathrm{O}$ with incremental number $2, \mathrm{C}=\mathrm{O}$ with 3 , while $\mathrm{O}-\mathrm{C}=\mathrm{O}$ with the number 4 respectively. Comparing the ratio of the oxydrilic bounds to the $\mathrm{C}-\mathrm{C}$ one, we can estimate the oxidation/reduction state. Comparing the panel a), b) and c) the GO reduction results evident, monitoring the progressive reduction of carboxylic groups.

The BP step behaves like a reducing agent improving also the simple UV-reduction. With Multipak software we are able to calculate the atomic percentage value of single bounds, reported on the left corner of the XPS plot. These values confirm the described behaviour.

From the ratio of $\mathrm{C}-\mathrm{C}$ versus oxidized groups (sum of peaks 2, 3 and 4) we obtain a value of:

- 1.2, for pristine GO (panel a),

- 3.3 for UV-exposed GO (panel b)

- 8.3 value for UV-exposed GO/BP (panel c).

The samples were functionalized with polymer after $90 \mathrm{~min}$ of UV irradiation. During the second reaction step. it is evident and increase of peaks " 2 ", " 3 " and "4" (panel d) compared to the RGO/BP (panel c), in samples where PEGMA monomers were grafted on the RGO/BP sheets (during the second reaction steps)the $\mathrm{C} 1 \mathrm{~s}$ peak evidences an increase of peaks " 2 ", " ' 3 "' and " 4 "' (panel d) in comparison to the RGO/BP of panel (c).

One possible explanation is that these peaks belong to the ether and acrylic groups present in the grafted chains. In order to have the possibility to check the grafting occurrence we choose heteroatoms with different shifted binding energy; respect to the Carbon/Oxygen bonds. The chosen heteroatoms adopted was fluorine for PFBA and nitrogen for DMAEM. The CF bond, if presents, is expected at 293 $\mathrm{eV}$. In panel e) is evident that grafting mechanism occurs, while, in the case of the functionalization with DMAEM, the presence of $\mathrm{CN}$ bonds could be associated to the strong increase of peak " 2 '" reported in panel (f). The presence of Nitrogen $1 \mathrm{~s}$ is confirmed also from the survey spectra (not shown), where a clear peak of nitrogen at $400 \mathrm{eV}$ is evident. The XPS data clearly demonstrated the reduction of GO occurs during the first irradiation step and the same analyses are able to confirm the efficiency of grafting during the second step. The grafting efficiency could be investigated by ATRIR analysis.

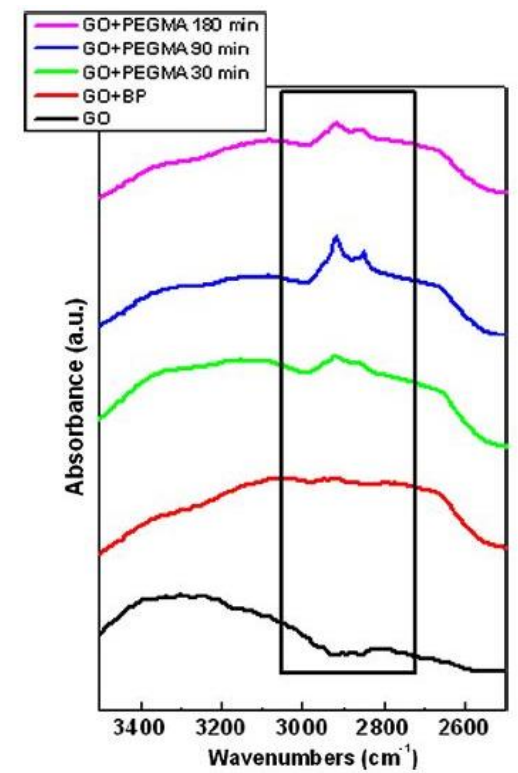

Fig. 5. ATR spectra of GO and UV modified GO, with BP and PEGMA with 30, 90, 180 min irradiation time.

In Fig. 5, it is reported, from the bottom, pristine $\mathrm{GO}$, GO treated with $\mathrm{BP}$ (from the first irradiation step) and the final modified-GO functionalized with PEGMA after 30, 90, $180 \mathrm{~min}$ irradiation times. A peaks at $2900 \mathrm{~cm}^{-1}$ (C-H stretching peaks) is presents in all spectra obtained by UV irradiation in presence of PEGMA, and it is attributable to the presence of grafted polymer chains, as a preliminary evidence of the grafting process efficiency.

\section{Conclusion}

In this paper, we have proposed two different fast and low cost methods for reduce GO in rGO embedded in polymeric matrices. The first innovative described method consists in a in situ reduction of GOx into an epoxy resin during its thermal curing in presence of amine. Optimizing the best curing conditions for the pristine epoxy resin, we observed a faster curing for the formulations containing GOx. The reduction of GOx was explained considering an enhancement of electrical conductivity for the filler system compared to the pristine resin. The second method was introduced to avoid the unique big temperature limitation of the first method. The reduction of GO in the second described method avoid thermal treatments. The UV improve the dispersion of rGO into organic solvents and polymeric matrices.

The first step of the process consists in the grafting of semipinacol groups onto GO surfaces. The rules of those act as photo initiator in a second step of functionalization in presence of acrylic/methacrylic monomers. The consequence is the formation of polymer chains grafted on the sheets surface by a "'grafting from' free radical polymerization process. The semipinacol grafting reduce GO in rGO with high electrical conductivity. This method gives us the 
possibility to a fast functionalization grafting a great variety of monomers. This procedure results appealing for the fabrication of polymer nanocomposites with possible application in coating and printable inks.

\section{Acknowledgements}

I would like to acknowledge:

- the (DISAT) PoliTo (Italy) head staff G.Saracco and coworkers: M. Castellino, G. Cicero, D. Foix, F. Giorgis, A. Tagliaferro;

- the IIT@ PoliTo (Italy) head staff C.F. Pirri and coworkers: K. Bejtka, A. Chiappone, A. Chiodoni, A. Chiolerio, R. Giardi, S. Porro, I. Roppolo. This work was founded by FFABR 2018.

\section{References}

1. Huang, Y.; Dong, X.; Shi, Y.; Li, C. M.; Li, L. J.; Chen, P.; Nanoscale, 2010, 2, 1485.

2. Yoo, E.; Kim, J.; Hosono, E.; Zhou, H.; Kudo T.; Honma, I.; Nano Lett., 2008, 8, 2277.

3. Soldano, C.; Mahmood, A.; Dujardin, E.; Carbon N. Y., 2010 , 48, 2127.

4. Sangermano, M.; Tagliaferro, A.; Foix, D.; Castellino, M.; Celasco, E.; Macromol. Mater. Eng., 2014, 299, 757.

5. Roppolo, I.; Chiappone, A.; Bejtka, K.; Celasco, E.; Chiodoni, A.; Giorgis, F.; Sangermano, M.; Porro, S.; Carbon N. Y., 2014, 77, 226.

6. Singh, S. K.; Singh, M. K.; Nayak, M. K.; Kumari, S.; Grácio, J. J. A.; Dash, D.; Carbon N. Y., 2011, 49, 684.

7. Zhu, Y.; Murali, S.; Cai, W.; Li, X.; Suk, J. W.; Potts, J. R.; Ruoff, R. S.; Adv. Mater., 2010, 22, 3906.

8. Celasco, E.; in Handbook of Graphene, Eds. Mishra, S.; Patra S.; Mishra, A.; WILEY-Scri., 2019.

9. Akhavan, O.; Ghaderi, E.; J. Phys. Chem. C, 2009, 113, 20214.

10. Williams, G.; Seger, B.; Kamat, P. V.; ACS Nano, 2008, 2, 1487.

11. Ding, Y. H.; Zhang, P.; Zhuo, Q.; Ren, H. M.; Yang, Z. M.; Jiang, Y.; Nanotechnology, 2011, 22, 215601.

12. Giardi, R.; Porro, S.; Chiolerio, A.; Celasco E.; Sangermano, M.; J. Mater. Sci., 2013, 48, 1249.

13. Stankovich, S.; Dikin, D. A.; Piner, R. D.; Kohlhaas, K. A.; Kleinhammes, A.; Jia, Y.; Wu, Y.; Nguyen, S. T.; Ruoff, R. S.; Carbon N. Y., 2007, 45, 1558.

14. Yang, D.; Velamakanni, A.; Bozoklu, G.; Park, S.; Stoller, M.; Piner, R. D.; Stankovich, S.; Jung, I.; Field, D. A.; Ventrice, C. A.; Ruoff, R. S.; Carbon N. Y., 2009, 47, 145. 